LBL-37732

UC-401

\title{
Chemical Reaction Dynamics Using the Advanced Light Source
}

\author{
X.Yang, ${ }^{\text {a D.A. Blank, }}$, J. Lin, ${ }^{*}$ P.A. Heimann, ${ }^{b}$ \\ A.M. Wodtke, ${ }^{c}$ Y.T. Lee, ${ }^{*}$ and A.G. Suits ${ }^{a}$ \\ Department of Chemistry, University of California, Berkeley \\ and \\ aChemical Sciences Division and bAccelerator and Fusion Research Division \\ Lawrence Berkeley Laboratory, University of California \\ Berkeley, California 94720 \\ cUniversity of California, Santa Barbara \\ Department of Chemistry \\ Santa Barbara, CA 93106
}

September 1995

*Present address: Institute of Atomic and Molecular Sciences, Academia Sinica, Taipei, Taiwan, ROC.

This work was supported by the Director, Office of Energy Research, Office of Basic Energy Sciences,

Chemical Sciences Division, of the U.S. Department of Energy under Contract No. DE-AC03-76SF00098.2 


\section{DISCLAIMER}

This report was prepared as an account of work sponsored by an agency of the Uinited States Government. Neither the United States Government nor any agency thereof, nor any of their employees, make any warranty, express or implied, or assumes any legal liabiity or responsibility for the accuracy, completeness, or usefulness of any information, apparatus, product, or process disclosed, or represents that its use would not infringe privately owned rights. Reference herein to any specific commercial product, process, or service by trade name, trademark, manufacturer, or otherwise does not necessarily constitute or imply its endorsement, recommendation, or favoring by the United States Government or any agency thereof. The views and opinions of authors expressed herein do not necescarily state or reflect those of the United States Government or any agency thereof. 


\section{DISCLAIMER}

Portions of this document may be illegible in electronic image products. Images are produced from the best available original document. 


\section{Chemical Reaction Dynamics Using The Advanced Light Source}

X. Yang, D. A. Blank, J. Lin a), P. A. Heimann, A. M. Wodtke, Y. T. Lee ${ }^{a)}$ and A. G. Suits Department of Chemistry

University of California,Berkeley

and

Chemical Sciences and Accelerator and Fusion Research Divisions

Lawrence Berkeley National Laboratory

Berkeley CA 94720

a) Present address: Institute of Atomic and Molecular Sciences, Academia Sinica, Taipei, Taiwan, $R O C$ 


\section{Abstract}

The recently commissioned Advanced Light Source (ALS) at Berkeley provides a high brightness, tunable VUV light source for chemical dynamics studies. A dedicated chemical dynamics beamline has been built at the ALS for studies of fundamental chemical processes. High flux ( $10^{16}$ photon/s with $2 \%$ bandwidth) VUV synchrotron radiation from 5 to $30 \mathrm{eV}$ can be obtained from the beamline, whose source is the U8/10 undulator. Three endstations will be in operation for studies ranging from crossed beam reaction dynamics and photodissociation to high resolution photoionization dynamics and spectroscopy. A rotatable source crossed molecular beam apparatus (endstation one) has been established for unimolecular and bimolecular reactive scattering studies. Photodissociation of methylamine and ozone were carried out using VUV synchrotron radiation as the ionization detection technique at this endstation. Results show the advantages of the new endstation using VUV ionization as the detection scheme over similar machines using electron bombardment as the ionization source. 


\section{(1) INTRODUCTION}

The studies of bimolecular and unimolecular reactions using molecular beam techniques have greatly improved our understanding of chemical reactions. The angular resolved time of flight (TOF) method for reactive scattering studies gives us the dynamical information that is needed to understand the detailed dynamics of reactive processes. ${ }^{1}$ Currently used universal TOF detection techniques for crossed beam reactive scattering studies are mostly associated with electron bombardment ionization. While electron bombardment ionization is the standard technique for mass spectrometric detection, it does have certain disadvantages, such as: extensive dissociative ionization, limited TOF resolution because of its finite ionization region, high background for certain masses, and certainly no selective ionization of different species.

Light, from the early flash lamp source to the modern lasers, has been widely used in the studies of chemical processes. Tunable VUV coherent light as the ionization detection technique in TOF measurements, instead of electrons, has tremendous advantages over the widely used electron bombardment ionization method. Firstly, tunable VUV ionization can be species selective since the ionization potentials for different species are different in nature. As a result it is also possible to eliminate the high background at mass $15\left(\mathrm{CH}_{3}{ }^{+}\right), 16\left(\mathrm{CH}_{4}{ }^{+}\right), 18$ $\left(\mathrm{H}_{2} \mathrm{O}^{+}\right), 28\left(\mathrm{CO}^{+}\right)$. This is particularly important when detecting radical species with $\mathrm{P}$ much lower (in many cases) than those of the background molecules. Tunable VUV light also allows us to obtain information about the internal energy of the reactive species due to the different characteristics of energy dependent ionization cross sections between "cold" and "hot" species. Secondly, soft (low energy) VUV ionization is expected to cause much less fragmentation than electron bombardment ionization, making the detection and analysis of multiple channel reaction processes simpler and more straightforward. Thirdly, since undulator VUV light is focusable, the ionization region can be much smaller than a comparable electron bombardment ionizer, making both the TOF and angular resolution much higher. Since VUV ionization is focusable with a relatively small associated heat load, the ionization region can also be easily cooled to very low temperature, making the background much smaller in the detector. However, in order to make the VUV ionization a standard universal detection technique for chemical reactions, one has to meet the following two requirements: high VUV photon flux (high enough sensitivity) and wide range tunability, which are hard to meet even by the modern state-of-art laser techniques. The recently developed third generation synchrotron radiation source at Berkeley, namely the Advanced Light Source, provides us such a light source. An undulator based VUV beamline has been constructed at the ALS, providing $\sim 10^{16}$ photon/s (2\% bandwidth) VUV light, tunable from 5 to $30 \mathrm{eV} .^{2}$ In addition to the full undulator light, 
high resolution VUV light is also available for the beamline, which can be used for high resolution studies on photoionization processes in the VUV region.

In this paper we will briefly describe the ALS chemical dynamics beamline and endstation one (rotating source cross beam machine), then present our first results using synchrotron radiation to study photodissociation of methylamine and ozone using endstation one.

\section{(2) EXPERIMENTAL SET UP}

\section{a) The ALS Chemical Dynamics Beamline}

The Advanced Light Source is one of the first third generation, low emittance, synchrotron radiation facilities. ${ }^{3}$ The ALS is designed based on insertion devices (undulators, wigglers) which produce tunable VUV to soft X-ray radiations with much higher photon flux than bending magnets. A U8 undulator, which will be replaced soon with a U10 undulator, is currently being used as the light source of the chemical dynamics beamline. The lowest energy achievable for the $\mathrm{U} 8$ undulator is $18 \mathrm{eV}$ for a $1.5 \mathrm{GeV}$ storage ring energy, and $8 \mathrm{eV}$ for a $1.0 \mathrm{GeV}$ storage ring energy. The future U10 undulator can produce VUV light as low as $5 \mathrm{eV}$ for a standard $1.5 \mathrm{GeV}$ storage ring energy.

- Figure 1 shows the layout for the chemical dynamics beamline. Light produced from the undulator is directed through horizontal and vertical apertures, whose openings are matched to the central cone size. The M1 and M2 mirrors absorb unwanted light at short wavelengths and focus the light at the center of the harmonic filter, which is developed to suppress the bigher harmonics of undulator radiation. A doubly differentially pumped scheme is used in the harmonic filter to fulfill the requirement of high number density ( $\sim 30$ torr) for the gas cell in order to achieve effective suppression while preserving the beamline high vacuum to $<5 \times 10^{-9}$ torr. It has achieved greater than $10^{4}$ suppression of the higher harmonics with no measurable $(<5 \%)$ attenuation of the fundamental. A detailed description of the instrument can be found in Ref. 4. After the harmonic suppressor, the VUV light is reflected by the retractable M3 mirror to endstation one, which is the rotating source crossed beam machine. M3 also focuses the light at the detection region of the crossed beam machine. An imaging endstation will be installed in front of endstation one in the near future, primarily for complementary crossed beam studies using the recently developed imaging technique.

If M3 is removed from the beam path, the VUV light will be reflected by M4 towards M5, which focuses the light onto the entrance slit of the 6.55 meter eagle monochromator, manufactured by McPherson, Corp. The achievable resolution of the monochromator will be 
$10^{5}$ or better, providing endstation two with an extremely high resolution VUV light source. After the monochromator, the light is then refocused by the M6 mirror into endstation two, primarily for various photoionization studies.

\section{b) The.Rotating Source Crossed Beam Endstation}

The newly built endstation one on the chemical dynamics beamline features the world's first crossed beam machine using synchrotron radiation as the detection method. Figure 2 shows the schematic of the crossed molecular beam apparatus. It has three main chambers: the rotating source, the main chamber and the detector. The rotating source has two main source chambers, each of which has a differentially pumped region. The two sources fixed at 90 degrees, and the whole source chamber is rotatable from -20 to 110 degrees (total 130 degrees rotation). The entire machine is pumped by oil-free magnetic bearing turbomolecular pumps. Each of the two main source region for the two sources is pumped by a high-throughput 2000 1/s turbopump (Seiko-Seiki STPH2000C), and each of the two differential pumping regions is pumped by a 300 or $400 \mathrm{l} / \mathrm{s}$ turbopump (Seiko-Seiki STP 300/400). The main chamber, which serves as the region where chemical reactions take place, is pumped by a $2000 \mathrm{1} / \mathrm{s}$ turbopump (Seiko-Seiki STP2000C). A base plate cooled by liquid nitrogen is also available for cryopumping of the main chamber. There are four regions in the detector. Region I, pumped by a $300 \mathrm{l} / \mathrm{s}$ turbopump, is the first differential region. Region II, also pumped by a $300 \mathrm{l} / \mathrm{s}$ pump, is the second differential region which also connects with the advanced light source. Region III is the ionization region, which includes a VUV ionizer, an electron bombardment ionizer and ion optics to transport ions. It is pumped by a $600 \mathrm{~J} / \mathrm{s}$ pump. Quadrupole mass selection (Extrel) then follows in region IV which also houses a standard Daly ion detector. Time of flight chopper wheels are available in front of the detector for velocity and crosscorrelation measurements.

Three types of experiments are possible at endstation one. a) Crossed molecular beam reactions, detected either by VUV ionization with mass selection, or electron bombardment ionization; b) Single molecular beam laser photodissociation experiments using the detection schemes same as a; c) Photodissociation by VUV synchrotron radiation experiments using electron bombardment ionization detection.

The first set of experiments carried out on the endstation one are focused on photodissociation of molecules by an excimer laser using tunable VUV ionization for detection. Only one molecular beam source is used in these experiments. An excimer laser is focused at the axis of rotation of the molecular beam source. Photodissociation products are then detected as a function of flight time and scattering angle. Results will be shown in the following section 
for methylamine photodissociation at $193 \mathrm{~nm}$ and ozone photodissociation at $193 \mathrm{~nm}$ and 248 nm.

\section{(3) RESULTS}

\section{a) Photodissociation of Methylamine}

Photodissociation study of hydrocarbons is an important topic since it helps us to understand the energetics and the dynamics of how these molecules break apart. It can also be used to measure the bond eneroies. In this experiment, the photochemistry of methylamine at $193 \mathrm{~nm}$ was studied using the angular resolved time of flight technique. Tunable VUV light of $8.5 \mathrm{eV}$ or higher was used in this experiment with a storage ring running at $1.0 \mathrm{GeV}$. Figure 3 shows the TOF spectrum of $\mathrm{m} / \mathrm{e}=30 / \mathrm{CH}_{3} \mathrm{NH}^{+}, \theta=12.5$ degree $(\theta$ is the angle between the molecular beam and the detector). This shows the hydrogen atom loss channel

$$
\mathrm{CH}_{3} \mathrm{NH}_{2}+h v \rightarrow \mathrm{CH}_{3} \mathrm{NH}+\mathrm{H}
$$

for methylamine at $193 \mathrm{~nm}$ photodissociation. Signal at $\mathrm{m} / \mathrm{e}=29$ was also observed, which was the strongest signal observed; however its TOF profile is distinctively different from that of $m / e=30$. The origin of the $m / e=29$ signal is not yet clear. There are three possibilities: a) the $m / e=29$ signal is from secondary dissociation of $m / e=30$ because of the unstable nature of $\mathrm{m} / \mathrm{e}=30 ; \mathrm{b}$ ) it is from a concerted $\mathrm{H}_{2}$ loss process; or c) both. Further analysis is needed in order to clarify the picture. Experimental measurements were also made to determine the relative ionization cross section for the $\mathrm{m} / \mathrm{e}=29$ product by tuning the undulator gap, shown in Figure 4. This type of data contains information on the internal excitation of the photofragment. However, in order to extract such information, one need to know the relative ionization cross section near the IP for the same species at low temperature. Accurate theoretical calculations are definitely required in this case.

Signal at $\mathrm{m} / \mathrm{e}=15,16$ are also observed from methylamine photodissociation at 193nm. Figure 5 shows the time of flight spectra of $m / e=15$ at $\theta=25$ degrees, and $m / e=16$ at the same angle. Eventhough this is a very minor channel (a few percent of the total dissociation), good signal to noise can still be obtained due to the extremely low background at both $\mathrm{m} / \mathrm{e}=15$ and 16. By tuning the VUV energy lower than the IP of the background gas, the background can be almost eliminated. Clearly, the $\mathrm{m} / \mathrm{e}=15,16$ signals are corresponding to the methyl group loss channel,

$$
\mathrm{CH}_{3} \mathrm{NH}_{2}+. h v \rightarrow \mathrm{CH}_{3}+\mathrm{NH}_{2}
$$

Figure 5 shows the momentum matched fits to both $\mathrm{m} / \mathrm{e}=15$ and $\mathrm{m} / \mathrm{e}=16$ at 25 . degrees using a single translational energy probability distribution. 
In conclusion, at least two dissociative channels have been observed for methylamine photodissociation at $193 \mathrm{~nm}$. These experimental results also show some of the advantages of VUV ionization over the filament ionization: much lower background and less dissociative ionization.

\section{b) Photodissociation of Ozone}

UV photodissociation of ozone is an important process in the upper atmosphere since it is the very process that protects living things from solar UV radiation. Recent studies show that a newly identified channel in the UV photodissociation of ozone might be responsible for a new ozone creation channel operating in the stratosphere, in addition to the established Chapman mechanism involving $\mathrm{O}_{2}$ photodissociation. ${ }^{5,6}$

In order to better understand the ozone problem, a series of experiments was carried out using endstation one with the storage ring running at $1.0 \mathrm{GeV}$. Figure 6 shows a series of TOF spectra, measured at $\mathrm{m} / \mathrm{e}=16$ and $\theta=30$ degree, with $193 \mathrm{~nm}$ as the photolysis source. In this experiment, the VUV light source is tunable from $8 \mathrm{eV}$. Therefore, the dependence of each photodissociation channel of product $\mathrm{O}_{2}$ on the ionization energy can be mapped out easily. One can see that individual peaks in the TOF spectrum change very differently as the ionization energy increases. This set of data contains information on how $\mathrm{O}_{2}$ is excited: vibrationally, electronically or both, since each type of excitation should show its unique characteristics of cross-section dependence on ionization energy. As all the electronic states of $\mathrm{O}_{2}$ below $8 \mathrm{eV}$ are known, it is possible through simulations to determine the state specific distribution of product $\mathrm{O}_{2}$. Similar experiments can be done for product $\mathrm{O}$ atom to identify the product state distribution of the $O$ atoms. This can be much simpler because atoms do not have rotational and vibrational states. Detail analyses and further experiments are underway in this direction.

Photodissociation of ozone at $248 \mathrm{~nm}$ has also been carried out using the full undulator light (without harmonic suppression) as the detection light source, with the fundamental at 18 $\mathrm{eV}$. In this experiment, a storage ring running at $1.5 \mathrm{GeV}$ is used with the $\mathrm{U} 8$ undulator. The lowest energy achievable is $18 \mathrm{eV}$, therefore tunable VUV selective ionization is not possible. Figure 7 shows the time of flight spectra of $\mathrm{O}_{2}$ and $\mathrm{O}$ when ozone was photolysed at $248 \mathrm{~nm}$. The data is not yet fully analyzed. Nevertheless this figure shows one of the main advantages of the new endstation: even at $18 \mathrm{eV}$ ionization energy there is little dissociative ionization in comparison with the previous results of photodissociation at $193 \mathrm{~nm}$ using electron bombardment ionization where dissociation ionization is extensive (Figure 4 in Ref. 5). Direct peak-to-peak momentum matching of the products can be seen, with little or no fragmentation of $\mathrm{O}_{2}$ to give mass 16 signal. Vibrationally resolved structures in the TOF spectra have been observed. 


\section{(4) CONCLUSTON}

A unique chemical dynamics beamline has been established for reaction dynamics studies at the Advanced Light Source. The new crossed beam endstation is now in full operation. Preliminary results are reported for photodissociation studies of methylamine and ozone. Experimental studies show that the new endstation using ALS VUV light as the ionization detection scheme has tremendous advantages over similar machines using electron bombardment ionization as the detection technique.

\section{ACKNOWLEDGMENT}

This work was supported by the Director, Office of Energy Research, Office of Basic Energy Sciences, Chemical Sciences Division, of the U.S. Department of Energy under contract no. DE-AC03-76SF00098. We also appreciate the excellent jobs done on the beamline by the LBNL engineering staff, and all the support from the ALS staff. 


\section{Figure Captions}

Figure 1. The chemical dynamics beamline layout.

Figure 2. Schematic of the rotating source crossed molecular beam endstation (endstation one).

Figure 3. Time of flight spectrum of methylamine photodissociation at $193 \mathrm{~nm}$ detected at $\mathrm{m} / \mathrm{e}=30, \theta=12.5$ degrees.

Figure 4. VUV ionization energy dependence of total ionization signal of the $m / e=29$ product at $\theta=12.5$ degree from photodissociation of methylamine at $193 \mathrm{~nm}$.

Figure 5. Time of flight spectra of $\mathrm{m} / \mathrm{e}=15,16$ at $\theta=25$ degrees, photon energy $13.0 \mathrm{eV}$. The open circles represent the experimental data. The solid line shows the fit to both spectra using a single translational energy probability distribution.

Figure 6. Time of flight spectra of $\mathrm{m} / \mathrm{e}=32$ from ozone photodissociation at $193 \mathrm{~nm}$ detected at $\theta=20$ degrees. Upper: ionization energy $17 \mathrm{eV}$, middle: $13.5 \mathrm{eV}$, lower: $10.5 \mathrm{eV}$. TOF peaks are labeled consistent with results from Ref. 5 .

Figure 7. Time of flight spectra of $\mathrm{m} / \mathrm{e}=16,32 \mathrm{from}$ ozone photodissociation at $248 \mathrm{~nm}$. 
References

${ }^{1}$ Y. T. Lee, Science 236, 793(1987).

${ }^{2}$ M. Koike, P. A. Heimann, A. H. Kung, T. Namioka, R. DiGennaro, B. Gee, N. Yu, Nuclear Instruments and Methods in Physics Reseach A 347, 282-286.

${ }^{3}$ A. S. Schlachter, New Directions in Research with Third-Generation Soft-Ray Synchrotron Radiation Sources, pp 1-22, NATO ASI Series E: Applied Sciences -Vol.254, edited by A. S. Schlachter and F. J. Wuilleumier, Kluwer Academic Publishers, 1992.

${ }^{4}$ A. G. Suits, P. A. Heimann, X. Yang, M. Evans, C. -W. Hsu, K.-T. Lu, A. H. Kung, and Y. T. Lee, Review of Scienctific Instruments, in press.

${ }^{5}$ Domenico Stranges, Xueming Yang, James D. Chesko, and Arthur G. Suits, J. Chem. Phys. 102(15), 1995.

${ }^{6}$ R. L. Miller, A. G. Suits, P. L. Houston, R. Toumi, J. A. Mack and A. M. Wodtke 265, 1831(1994). 




Figure 1 


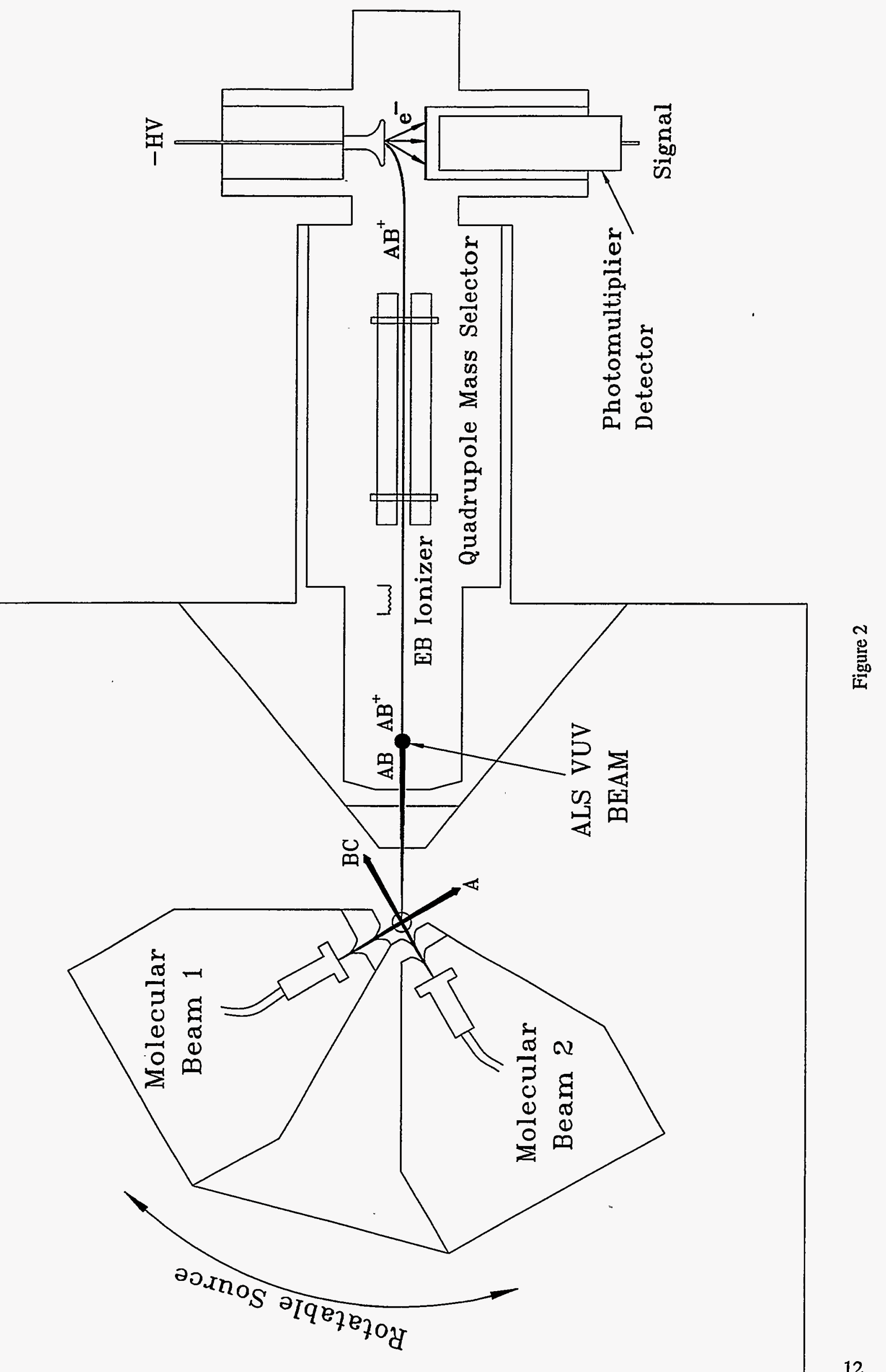




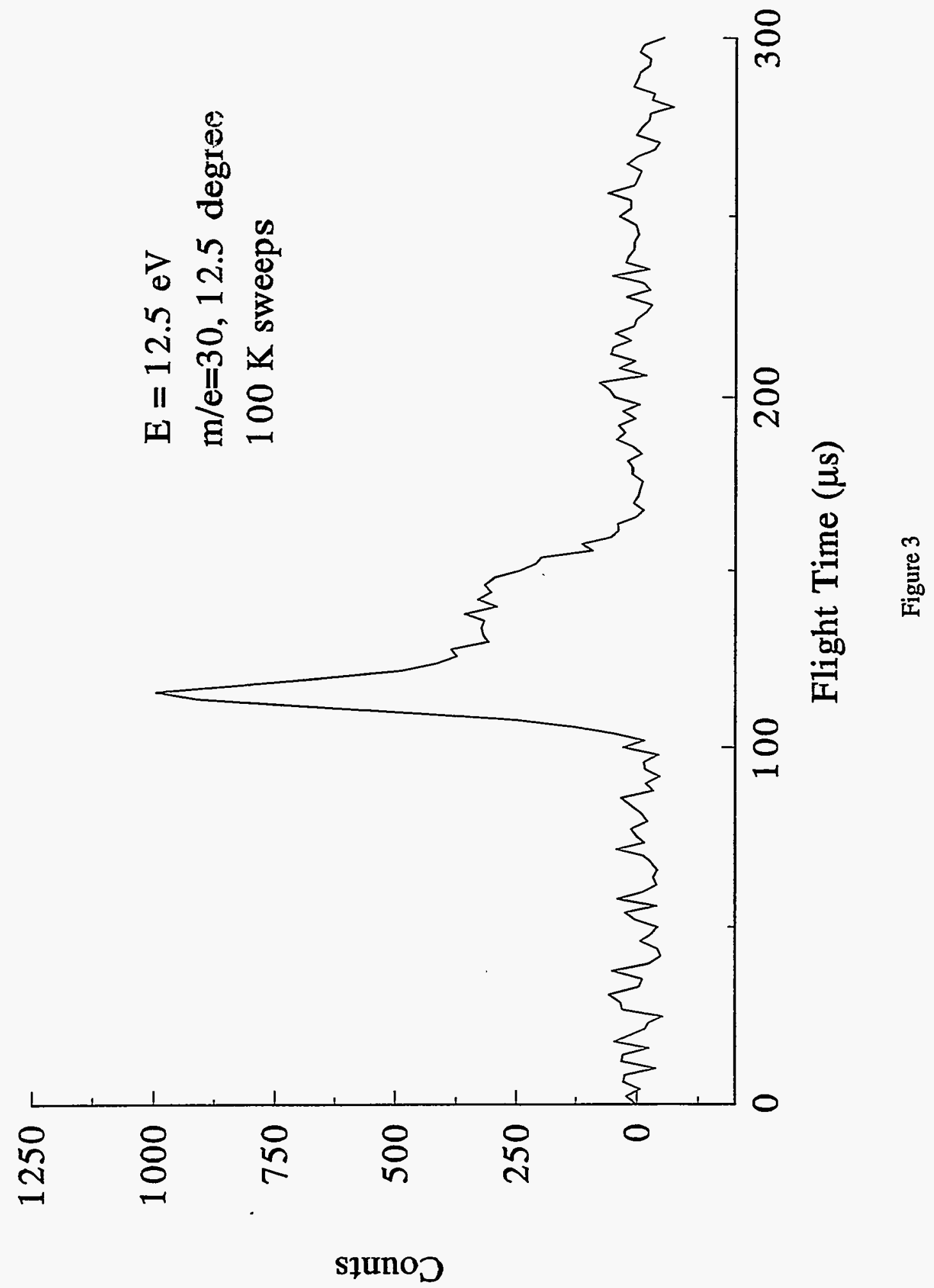




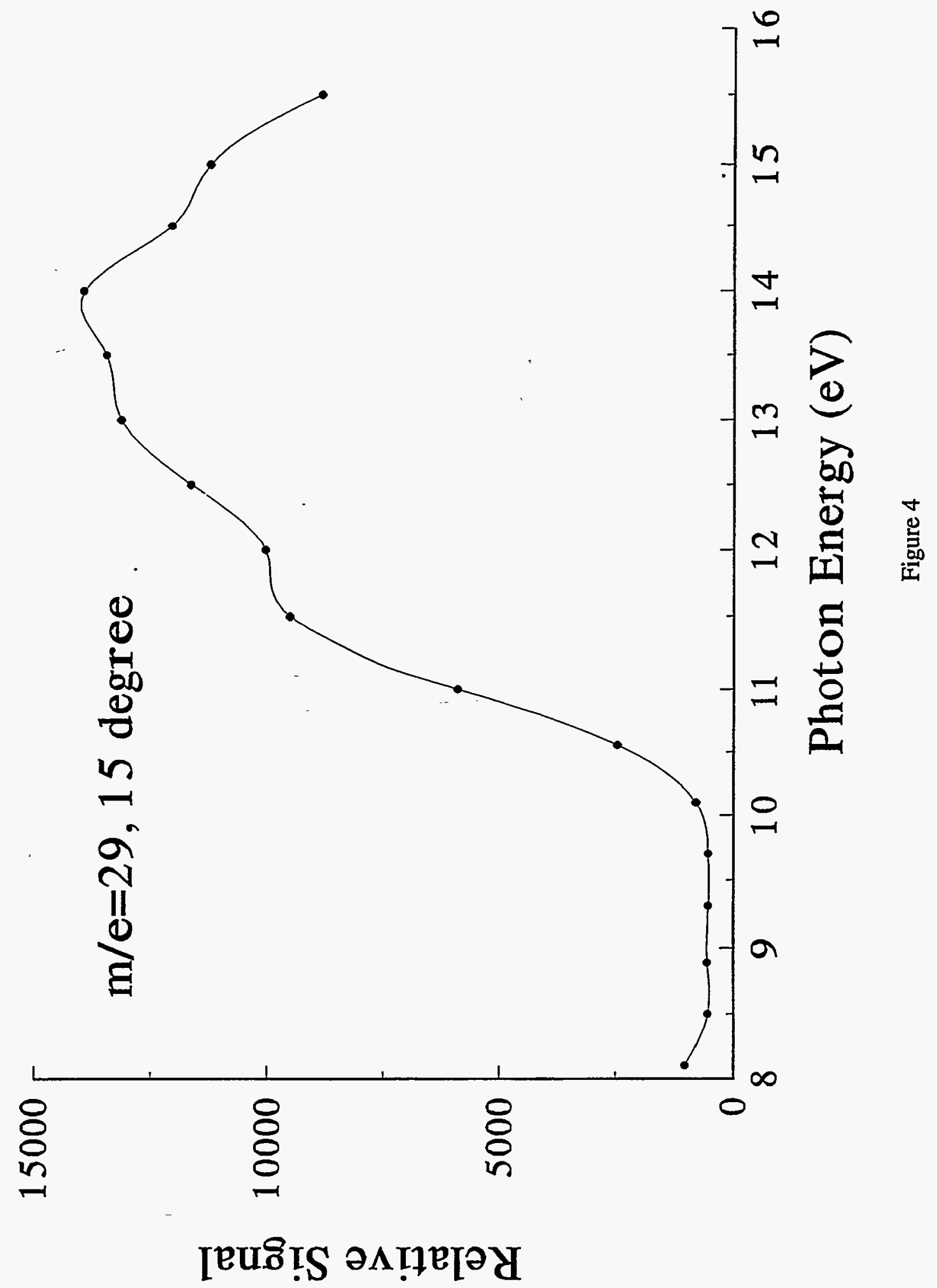



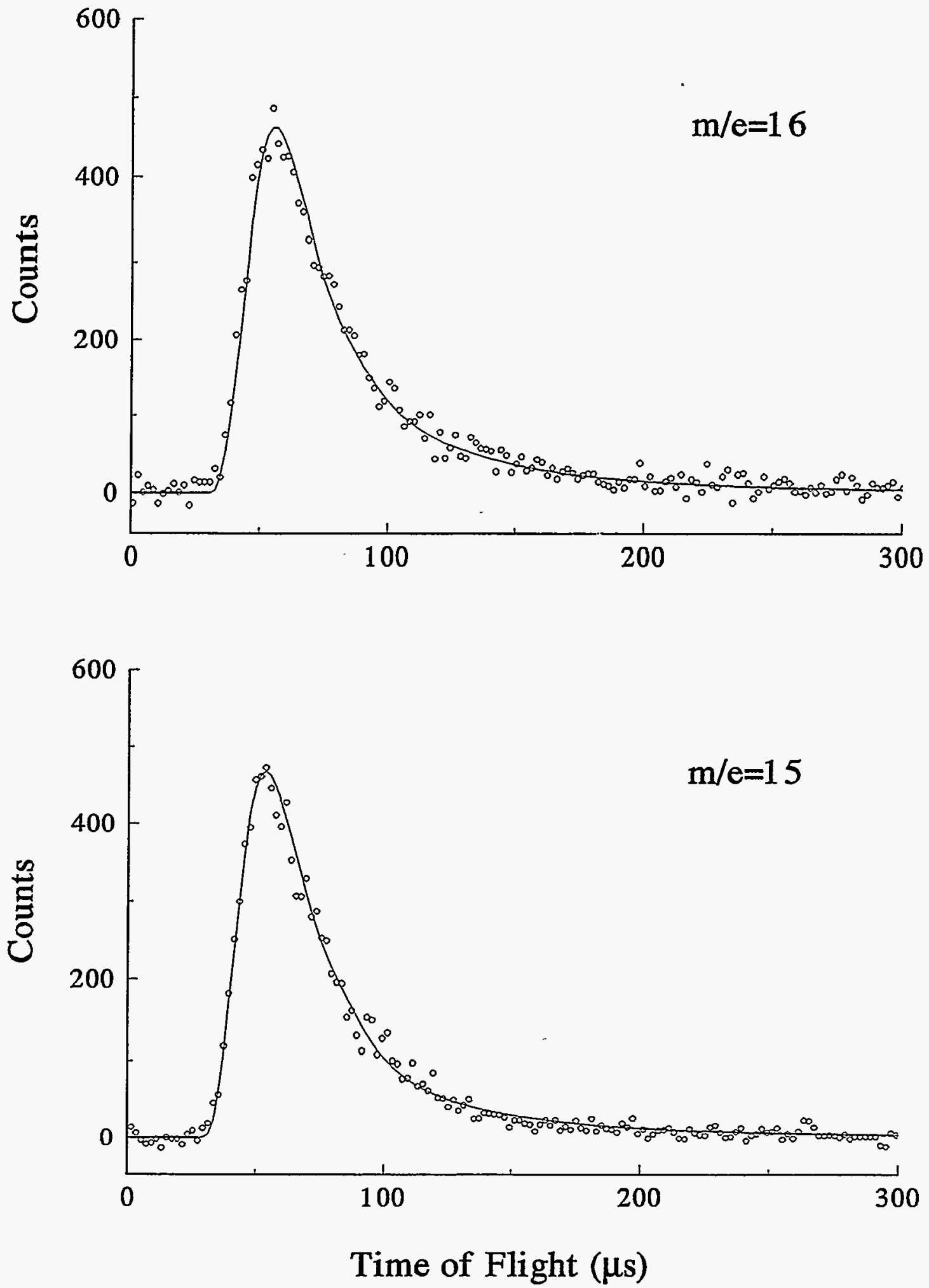

Figure 5 


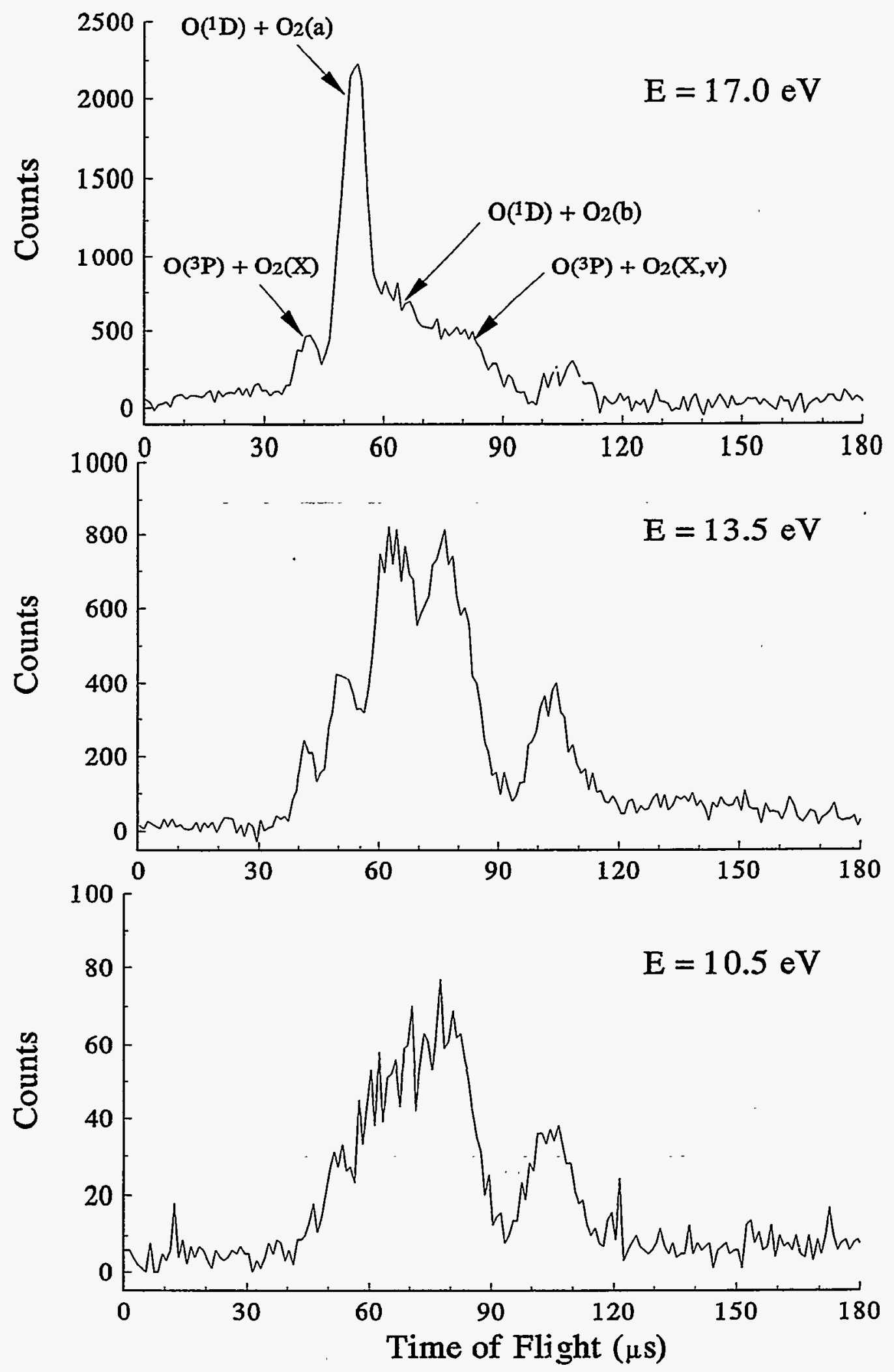

Figure 6 

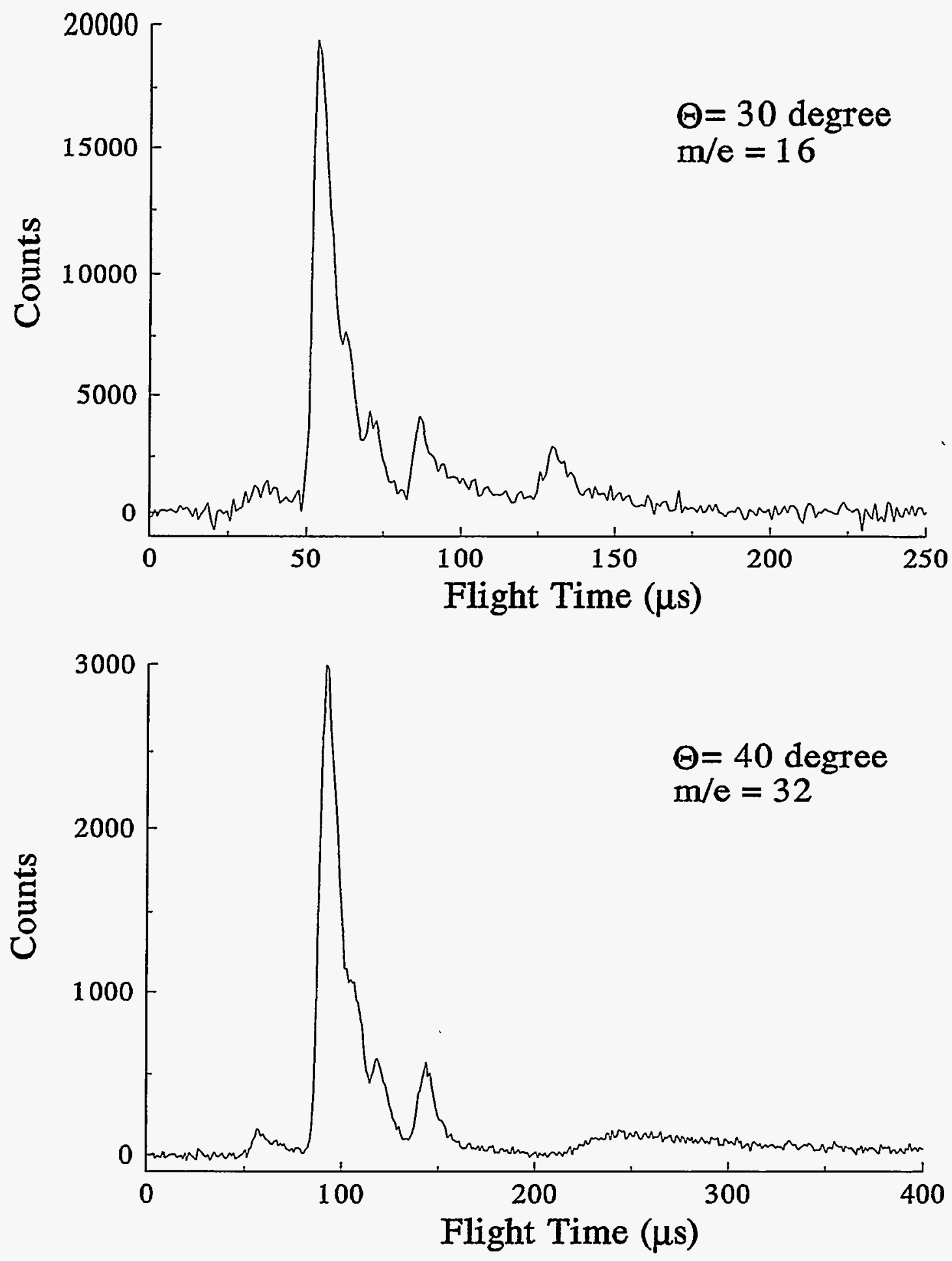

Figure 7 